\title{
Die häufigsten Neoplasien der Haut beim Hund - Ein Überblick
}

Julia Buchholz

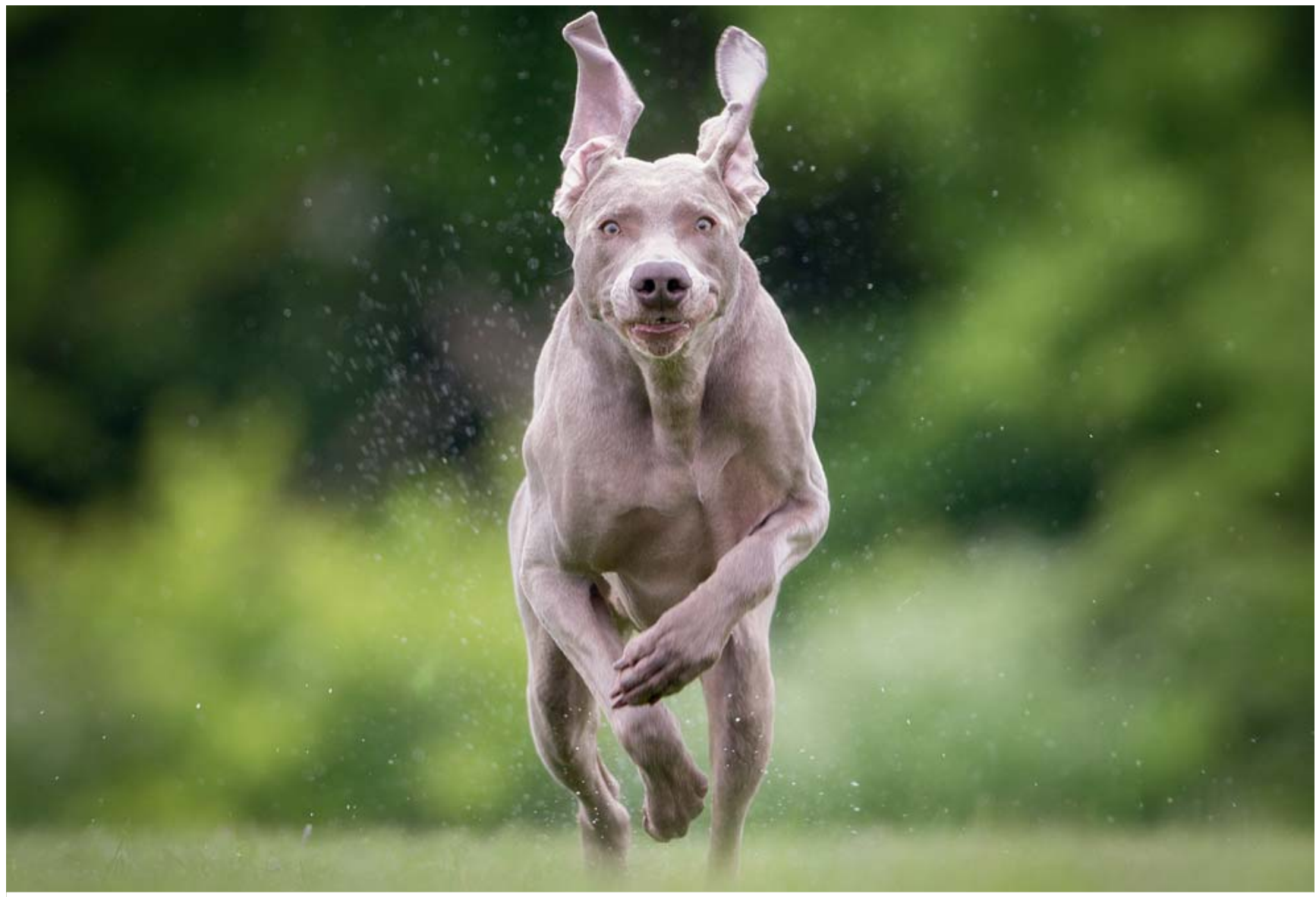

(c) Mikkel Bigandt - stock.adobe.com

Etwa $1 / 3$ aller Neoplasien des Hundes sind Hauttumoren und ca. 20-40\% dieser Tumoren sind bösartig. Doch welchen prognostischen Unterschied machen Ihre Diagnostik und die Therapieauswahl?

Die Haut ist das Organ, das beim Hund am häufigsten von Tumoren betroffen ist. Dies lässt sich dadurch erklären, dass die Haut als das größte Organ des Körpers sehr vielen Umwelteinflüssen - z. B. UV-Strahlung, Toxinen und viralen Faktoren - ausgesetzt ist. Der Tumorentstehung liegen auch genetische Faktoren und molekulare Mechanismen zugrunde, die in Zukunft in der Veterinärmedizin genauer erforscht werden müssen.

\begin{abstract}
Merke
Circa $1 / 3$ aller Neoplasien des Hundes sind Hauttumoren und etwa $20-40 \%$ dieser Tumoren sind bösartig.
\end{abstract}

Gelegentlich können kutane Massen Metastasen von anderen Tumoren darstellen.

Die Klassifizierung von Hauttumoren erfolgt anhand folgender Kriterien: 
- Ursprungsgewebe

- Ursprungszelle

- Malignitätsgrad

\section{Häufigste Hauttumoren beim Hund}

Die häufigsten Hauttumoren beim Hund sind:

- Mastzelltumor

- Plattenepithelkarzinom

- Melanom

- Fibrosarkom

- Hämangioperizytom

- Kutanes Lymphom

- Papillom

- perianales Adenom

- Lipom

- Talgdrüsenadenom/-hyperplasie

- Histiozytom

- Basalzellkarzinom

Die fett gedruckten Tumoren stellen die am häufigsten in der Onkologie vorgestellten Hautneoplasien dar. Die restlichen erwähnten Neoplasien können i.d. R. einfach kontrolliert werden. Dies erfolgt teilweise durch eine spontane Remission (z. B. beim Papillom und Histiozytom) oder durch eine marginale chirurgische Entfernung.

\section{Erscheinungsbild}

Verglichen zu Tumoren an anderen Lokalisationen können bei Hauttumoren die von außen sichtbaren Veränderungen vom Besitzer leichter entdeckt werden. Gutartige Tumoren wachsen i.d.R. langsamer, sind tendenziell eher nicht schmerzhaft und gut verschieblich. Bösartige Tumoren wachsen häufig schneller und sind eher mit den unterliegenden Strukturen verwachsen. Natürlich sind diese keine definitiven Kriterien und z.B. Ulzerationen können sowohl bei benignen als auch bei malignen Neoplasien beobachtet werden.

\section{Diagnostisches Vorgehen}

Eine detaillierte Anamnese und klinische Untersuchung sind essentiell. Des Weiteren sollten Umfangsvermehrungen nach folgenden Kriterien beurteilt werden:

- Lokalisation

- Größe

- Verschieblichkeit

- Vorhandensein von Ulzerationen

- ggf. Vorhandensein von Juckreiz

Eine einfache, schnelle und in den meisten Fällen ohne Sedation oder Narkose durchführbare diagnostische Maßnahme ist eine Zellenentnahme mittels Feinnadelaspiration (FNA), die häufig diagnostisch ist. Für Tumoren, die eine geringere Tendenz zur Exfoliation von Zellen haben (z.B. gut differenzierte Sarkome) kann allerdings eine Biopsieentnahme erforderlich sein. Eine FNA der regionalen Lymphknoten kann zum gleichen Zeitpunkt durchgeführt werden. Weitere notwendige diagnostische Abklärungen und Therapieoptionen sind abhängig vom Tumortyp ( $\triangleright$ Abb. 1).

\begin{abstract}
- Merke
Jede Masse, die chirurgisch entfernt wird, sollte zur histologischen Untersuchung eingeschickt werden, um das weitere Vorgehen und die Prognose bestimmen zu können.
\end{abstract}

\section{Mastzelltumoren}

Mastzelltumoren repräsentieren 16-21\% der kaninen Hauttumoren. Hunde jeden Alters können betroffen sein und es besteht eine Rasseprädisposition für folgende Hunderassen:

- Beagle

- Boxer

- Boston Terrier

- Golden Retriever

- Labrador Retriever

- Schnauzer

- Shar Pei

- Staffordshire Bull Terrier

- Weimaraner

Beim Boxer sind die Mastzelltumoren tendenziell weniger aggressiv, während Shar Peis eher aggressive Mastzelltumoren entwickeln. Zusätzlich gibt es Lokalisationen, in denen sich Mastzelltumoren eher aggressiv verhalten (v.a. perianal, präputial, inguinal, mukokutan, im Kopfbereich und Nagelbett). Die Ätiologie der Mastzelltumoren ist nicht bekannt, aber chronische Hautentzündungen stellen möglicherweise einen prädisponierenden Faktor dar.

\section{Erscheinungsbild und biologisches Verhalten}

Mastzelltumoren haben ein ausgesprochen vielfältiges Erscheinungsbild und können viele andere Hautveränderungen vortäuschen. Auch ihr biologisches Verhalten ist sehr variabel. Alle Mastzelltumoren verhalten sich in verschiedenem Ausmaß lokal invasiv. Circa 1/3 der Mastzelltumoren zeigt aggressives Verhalten mit Metastasierung in die regionalen Lymphknoten, Fernorgane (Leber, Milz, Knochenmark) oder Haut.

\section{Merke}

Einige Mastzelltumoren sehen aus wie Lipome - hier kann eine FNA der schnellen Differenzierung dienen.

\section{Paraneoplastische Symptome}

Im Falle einer Degranulation der in den Mastzellen vorhandenen intrazytoplasmatischen Granula (biologisch aktive Moleküle wie Proteasen, Zytokine, Heparin und Histamin) kann es zu lokalen Wundheilungsstörungen, 


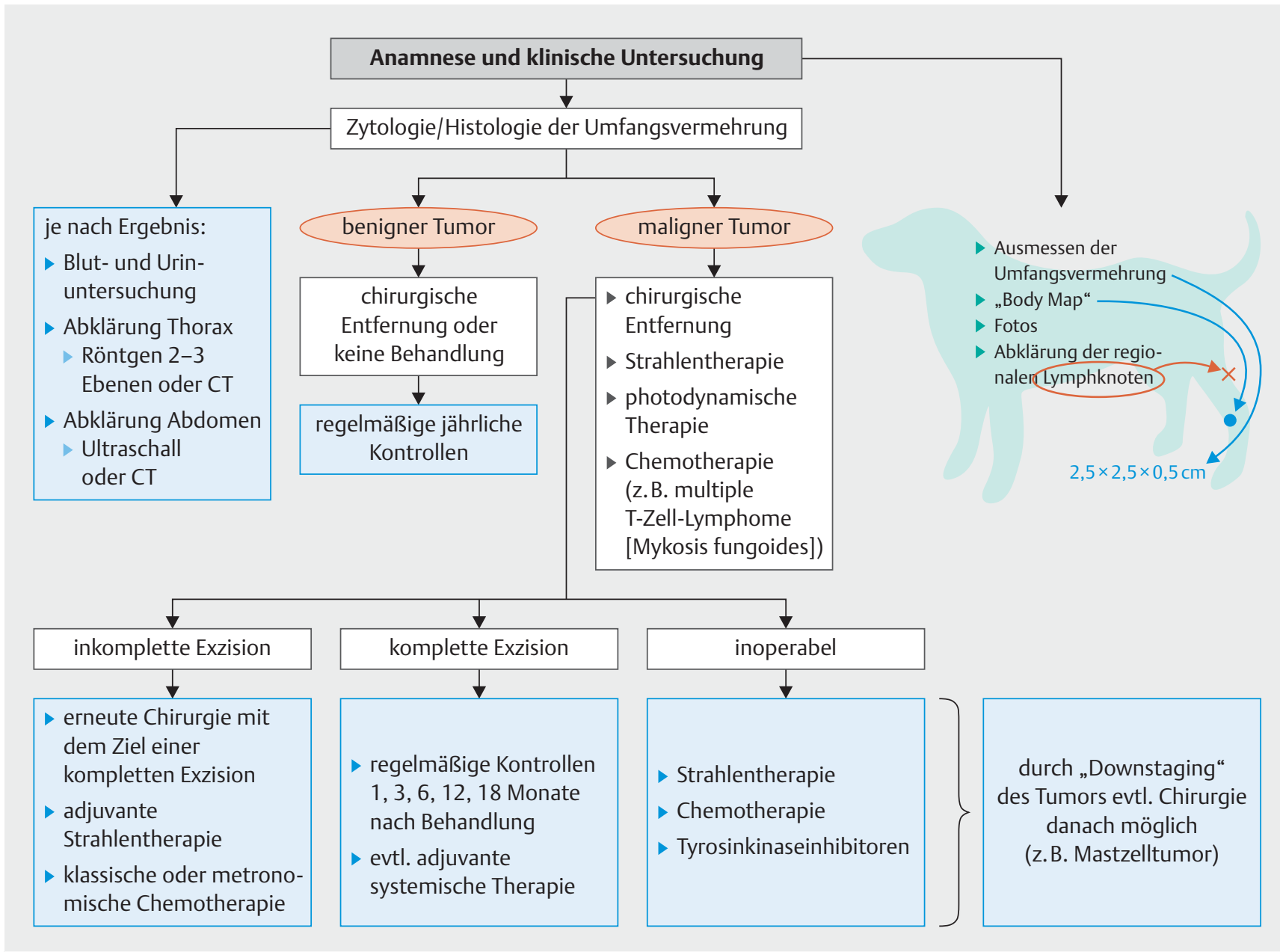

- Abb. 1 Diagnostisches und therapeutisches Vorgehen bei kaninen kutanen Neoplasien. @ Julia Buchholz

Blutungsneigung und Juckreiz kommen. Eine Degranulation kann spontan oder nach einem Trauma erfolgen.

\section{Merke}

Potentielle Mastzelltumoren sollten bei FNA, Biopsieentnahme und chirurgischer Exzision möglichst wenig manipuliert werden.

Häufig kommt es nach FNA zu Blutungen im Bereich des Einstichs und die Masse kann kurzfristig z. T. deutlich größer werden. Kommt es nach Manipulation eines Mastzelltumors zu einer Erythembildung und Kreisformation um den Tumor, spricht man vom „Darier's sign“ (Darier’sches Zeichen). Eine systemische Absorption von Histamin kann paraneoplastisches Erbrechen sowie Magen- und Darmulzerationen mit resultierender Melena hervorrufen. Selten kann es zu klinisch relevanter Hypotension kommen.

Die Wahrscheinlichkeit des Auftretens der beschriebenen paraneoplastischen Symptome ist umso höher, je schlechter differenziert der Tumor ist. Hochgradige Mastzelltumoren (Grad III nach Patnaik) haben eine höhere Tendenz, schnell zu wachsen, zu ulzerieren und Metastasen zu bilden. Gut differenzierte Mastzelltumoren bestehen z.T. seit Monaten, wachsen eher langsam und können sich haarlos präsentieren.

\section{Diagnostik}

Eine FNA ist bei einem Großteil der Mastzelltumoren diagnostisch: Typisch sind „metachromatisch“ anfärbende Granula, die jedoch bei schlecht differenzierten Mastzelltumoren fehlen und dadurch die Diagnose erschweren können. Die prognostisch wichtige Einteilung in Tumorgrade (Grad I-III nach Patnaik oder Grad I-II nach Kiupel, die biologische Aggressivität nimmt mit ansteigendem Grad zu) lässt sich jedoch nur histologisch durchführen. Bei der FNA der regionalen Lymphknoten muss man daran denken, dass diese eine hohe Anzahl normaler Mastzellen enthalten können. 

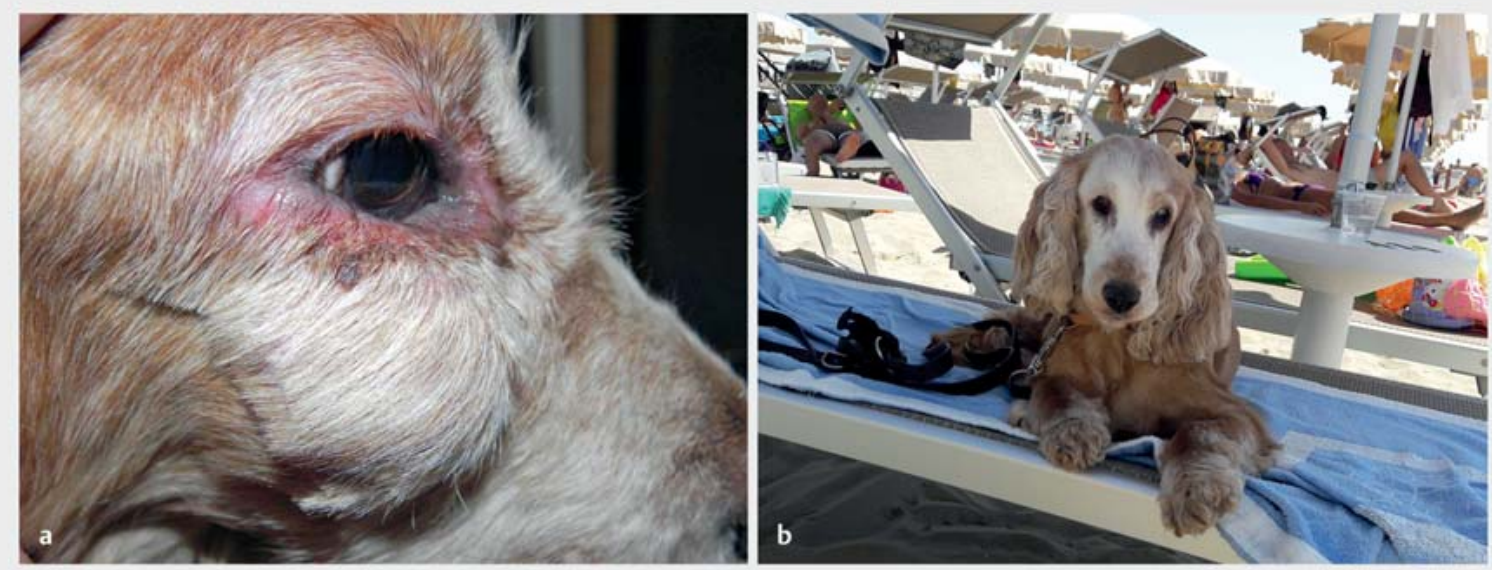

- Abb. 2 a Hündin mit rezidiviertem Grad II Mastzelltumor unter dem rechten Auge. Der Tumor zeigte kein Ansprechen auf die Chemotherapie und wurde anschließend palliativ bestrahlt. b Situation 4 Jahre nach der Bestrahlung: Der Tumor ist nach wie vor in kompletter Remission. () Julia Buchholz

\section{Prognostische Faktoren}

Das Auftreten multipler Mastzelltumoren ist nicht zwangsläufig als negativer prognostischer Faktor zu werten. Vielmehr spielt der histologische Grad jedes einzelnen Mastzelltumors eine wichtige prognostische Rolle. Eine zusätzliche wichtige Information im histologischen Bericht ist der mitotische Index sowie eventuell immunhistologische Angaben wie Ki67 und c-kit.

\section{Therapie}

Die Therapie der Wahl ist eine großzügige chirurgische Exzision. Eine Vorbehandlung mit Antihistaminika (Diphenhydramin $1-2 \mathrm{mg} / \mathrm{kg} 1 \mathrm{Mal} / \mathrm{Tag}$ am Abend) und Ranitidin ( $2 \mathrm{mg} / \mathrm{kg} 2 \mathrm{Mal} / \mathrm{Tag}$ ) kann potentiellen, durch Degranulation hervorgerufenen Nebenwirkungen vorbeugen. Ein lateraler Rand von $2 \mathrm{~cm}$ und eine En-BlocResektion in die Tiefe inklusive der Faszie ist für gut bis mittelgradig differenzierte (Grad I und Grad II nach Patnaik) Mastzelltumoren als kurativ anzusehen. Bis zu 30\% der gut differenzierten Mastzelltumoren mit histologisch knappen Rändern rezidiviert nicht. Bei den sich aggressiver verhaltenden Grad III Mastzelltumoren sollte lateral ein Rand von $3 \mathrm{~cm}$ entfernt werden, die Resektion in die Tiefe sollte erneut inklusive der Muskelfaszie erfolgen.

Die Strahlentherapie wird meist adjuvant zur Chirurgie (wenn der Tumor nicht mit sauberen Rändern entfernt werden konnte) eingesetzt. Für Mastzelltumoren, die nicht chirurgisch entfernbar sind oder nach Chirurgie rezidivieren, kann die Strahlentherapie auch als einzige Therapieform eingesetzt werden ( $\bullet$ Abb. 2).

Merke

Mastzelltumoren sprechen oft sehr gut auf eine Strahlentherapie an.
Die Chemotherapie wird auch meist adjuvant und hauptsächlich bei höhergradigen Mastzelltumoren eingesetzt. Wie auch die Strahlentherapie kann sie im Sinne einer Tumorverkleinerung (Downstaging) vor einer lokalen Therapie oder als rein palliative Maßnahme als einzige Therapieform eingesetzt werden. Am besten beschrieben und als erste Wahl anzusehen sind Protokolle, die auf Vinblastin/Prednisolon (Vinblastin $2 \mathrm{mg} / \mathrm{m}^{2}$ und Prednisolon $0,5-1 \mathrm{mg} / \mathrm{kg}$ ) und Lomustin (CCNU $70 \mathrm{mg} / \mathrm{m}^{2}$ ) basieren. Häufig kommen auch Tyrosinkinase-Inhibitoren wie Masitinib (Masivet ${ }^{\circledR} 12,5 \mathrm{mg} / \mathrm{kg} / \mathrm{Tag}$ ) oder Toceranib (Palla$\mathrm{dia}^{\circledR} 3,25 \mathrm{mg} / \mathrm{kg}$ jeden 2. Tag) zum Einsatz.

Bei Patienten mit multiplen Tumoren niedrigen Grades ist eine chirurgische Entfernung aller Massen empfohlen. Um neuen Tumoren vorzubeugen oder falls bei einem Patienten nicht alle Tumoren chirurgisch entfernt werden können, kann eine orale Chemotherapie mit Chlorambucil (5 mg/m² jeden 2. Tag) und Prednisolon $\left(20 \mathrm{mg} / \mathrm{m}^{2}\right.$ jeden 2. Tag) eingesetzt werden.

\section{Plattenepithelkarzinome}

Plattenepithelkarzinome können sowohl auf der Haut als auch an den Zehen vorkommen. Häufig wachsen sie flach und ulzerativ, sie können sich jedoch auch blumenkohlartig über die Hautoberfläche erheben.

\section{Nasenspiegel}

Die Plattenepithelkarzinome im Bereich des Nasenspiegels können sich beim Hund therapeutisch problematisch darstellen. Die Diagnose erfolgt meist mittels Biopsie, da eine Beurteilung der FNA durch die häufig assoziierte Entzündung erschwert sein kann. Die regionalen Lymphknoten sollten mittels FNA abgeklärt werden und 
die Lunge sollte anhand von Röntgenbildern (mind. 2 Ebenen) untersucht werden.

Im Gegensatz zur Katze sprechen diese Tumoren beim Hund nicht gut auf eine Strahlentherapie an und eine chirurgische Entfernung ist daher vorzuziehen (wenn auch ästhetisch z. T. weniger ansprechend). Wenn ein chirurgischer Eingriff für die Tierbesitzer nicht infrage kommt, kann alternativ eine Therapie mit Toceranib (Palladia ${ }^{\oplus}$, $3,25 \mathrm{mg} / \mathrm{kg}$ jeden 2. Tag) in Erwägung gezogen werden, da das Ansprechen vor allem bei ulzerierten Tumoren gut sein kann.

\section{Zehenbereich}

Das Plattenepithelkarzinom der Zehen ist mit knapp 50\% der häufigste in diesem Bereich vorkommende Tumor. Bei ca. 3\% der Hunde mit Plattenepithelkarzinomen der Zehen sind mehrere Zehen betroffen.

Häufig betroffene Hunderassen sind:

- Pudel

- Labrador Retriever

- Riesenschnauzer

- Gordon Setter

- Rottweiler

- Dackel

- Flat Coated Retriever

Sinnvoll zur diagnostischen Abklärung sind eine Biopsie der veränderten Läsion sowie lokale Röntgenbilder (in 2 Ebenen). Die regionalen Lymphknoten sollten mittels FNA abgeklärt werden. Bei Diagnose eines Plattenepithelkarzinoms sollte zusätzlich die Lunge untersucht werden (Röntgen oder CT).

Differenzialdiagnostisch sind außer dem Melanom (s.u.) auch diverse nicht neoplastische Veränderungen im Bereich der Zehen zu beachten, wie z. B. pyogranulomatöse Entzündungen, epitheliale Zysten, intrakutane verhornende Epitheliome etc.

Zum Zeitpunkt der Diagnose von Plattenepithelkarzinomen im Zehenbereich haben knapp 10\% der Hunde Metastasen, zusätzliche $23 \%$ entwickeln nachfolgend Metastasen. Auf lokalen Röntgenbildern zeigen sich in $80 \%$ der Fälle Knochenlysen.

Die Chirurgie ist auch hier die Therapie der Wahl. In den meisten Fällen kann eine weite chirurgische Entfernung mit einer Zehenamputation erreicht werden. Wenn danach die histologischen Ränder nicht tumorfrei sind, sollte eine Strahlentherapie angeschlossen werden, um eine Gliedmaßenamputation zu vermeiden. Die Überlebensraten nach Chirurgie betragen 50-83\% nach einem Jahr und $18-62 \%$ nach 2 Jahren.
NODULÄRE DERMATOFIBROSE

Bei Hunden, die mit multiplen Hautknoten vorgestellt werden, sollte auch an das mögliche Vorliegen einer nodulären Dermatofibrose gedacht werden. Diese Patienten leiden i.d. R. am renalen Zystadenokarzinom, klinisch relevant sind anfangs jedoch häufig die Hautveränderungen. Dieses Syndrom kommt vor allem beim Deutschen Schäferhund vor. Die Hautläsionen sollten chirurgisch entfernt werden. Meist sterben diese Hunde an einer Niereninsuffizienz oder einem progressiven Zystadenokarzinom im Durchschnitt ca. 3 Jahre nach der Diagnose. Zurzeit gibt es keine effektive Therapie.

\section{Melanome}

\section{Lokalisation und biologisches Verhalten}

Melanome können auf der Haut, an den Zehen und im Bereich der Mundschleimhaut vorkommen. Sie können sowohl gutartig als auch bösartig sein: Melanome der behaarten Haut sind nur zu 25-50\% bösartig, die im Bereich der Maulhöhle und Zehen deutlich häufiger.

Maligne Melanome neigen zur Metastasierung in Lymphknoten, Lunge und Bauchhöhlenorgane. Zum Zeitpunkt der Diagnose hat knapp 30\% der Hunde mit einem Melanom im Zehenbereich regionale Metastasen oder Fernmetastasen. Zusätzlich entwickeln knapp 40\% zu einem späteren Zeitpunkt Metastasen. Nur ca. 20\% zeigt auf lokalen Röntgenbildern eine Knocheninvasion [1]. Eine andere Studie konnte hingegen bei allen untersuchten Hunden ( $n=14$ ) histologisch eine Knocheninvasion feststellen [2]. Die Sensitivität für Knochenzerstörung ist beim Röntgen bekanntlich nicht allzu hoch.

\section{Diagnostik}

Die Diagnose erfolgt mittels FNA, Biopsie oder nach erfolgter chirurgischer Exzision.

\section{Therapie}

Die Therapie der Wahl ist die chirurgische Entfernung des Tumors. Die Überlebensraten ein Jahr nach der Chirurgie liegen bei 52-57\%, 2 Jahre nach dem Eingriff bei 13-36\%. Auch hier sollte bei inkompletter Tumorentfernung eine adjuvante Strahlentherapie in Erwägung gezogen werden. Melanome werden aus radiobiologischen Gründen hypofraktioniert (mit einer hohen Dosis pro Bestrahlungsfraktion) bestrahlt. Häufig sieht man auch bei Bestrahlung makroskopischer Melanome ein Ansprechen, eine chirurgische Entfernung sollte dennoch wenn immer möglich vorausgehen.

Aufgrund der hohen Metastasierungsrate sollte der Einsatz einer systemischen adjuvanten Therapie mit dem 

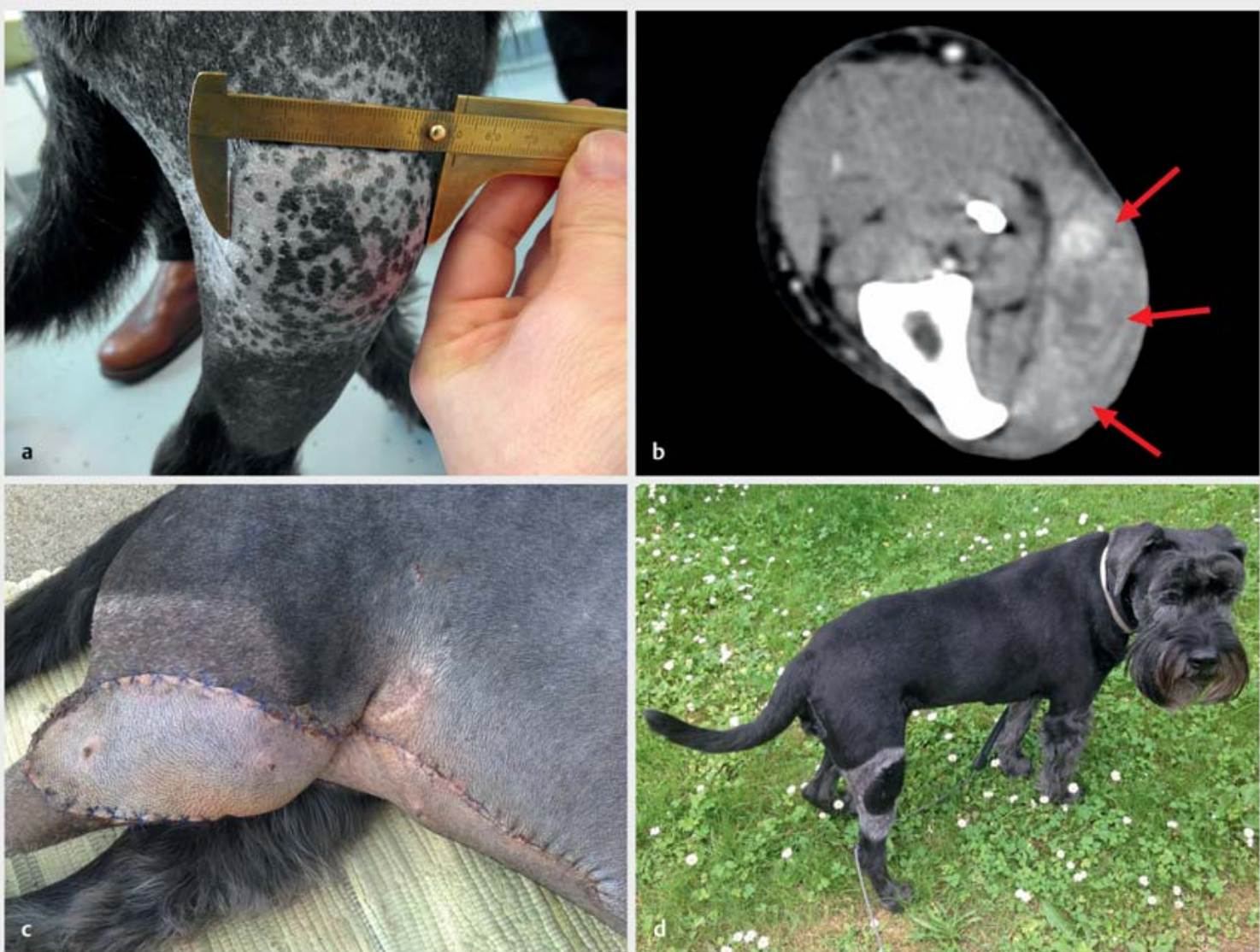

- Abb. 3 a Hündin mit Hämangioperizytom 2 Monate nach kurativer Bestrahlung. Der Tumor ist besser begrenzt und kleiner als vor der Bestrahlung. b CT-Bild 4 Monate nach der Bestrahlung zur Beurteilung einer eventuellen Chirurgie. c Der Tumor wurde 4,5 Monate nach der Bestrahlung chirurgisch entfernt. Die seitlichen Ränder waren sauber, in die Tiefe waren die Ränder knapp. Der Defekt wurde mittels eines „caudal superficial epigastric Skin Flap“ gedeckt. $\mathbf{d}$ Situation 6 Monate nach der Chirurgie. Die Patientin lebte noch 2,5 Jahre und starb nicht an ihrer Tumorerkrankung. a, b und d @ Julia Buchholz, c @ Sandra Schallberger

Besitzer besprochen werden. Da Chemotherapien keinen erwähnenswerten Erfolg zeigten und da es sich beim Melanom um einen immunogenen Tumor handelt, kommen adjuvant vor allem Immuntherapien infrage. Die Verwendung von Impfungen (z. T. individuell hergestellt mithilfe tumoreigenen Materials) ist beschrieben und wird teilweise routinemäßig eingesetzt. Auch der Einsatz von Mistelpräparaten ist beim Melanom sinnvoll (z. B. Iscador $^{\circledR} \mathrm{P}$, subkutane Applikation durch den Besitzer $3 \mathrm{Mal} /$ Woche).

\section{Weichteilsarkome}

Diese Gruppe maligner Tumoren ist mesenchymalen Ursprungs. Die Bezeichnung des Tumors leitet sich aus dem Ursprungsgewebe ab, dies kann z. B. das Bindegewebe (Fibrosarkom) oder das Blutgefäßendothel (Hämangioperizytom/Hämangiosarkom) sein.

\section{Biologisches Verhalten}

Weichteilsarkome wachsen stark infiltrativ in das umliegende Gewebe hinein. Die Metastasierungstendenz ist generell eher gering, hängt vom histologischen Grad ab (Grad I: 10\%, Grad II: 20\%, Grad III: 42\%) und erfolgt vor allem in Lunge und Lymphknoten.

\section{Diagnostik}

Eine FNA ist beim Sarkom häufig nicht diagnostisch, da diese Tumore eine geringere Tendenz zur Exfoliation von Zellen haben. Daher ist eine inzisionale Biopsie zur Diagnosestellung zu empfehlen.

\section{Therapie}

Die Behandlung der Wahl ist eine chirurgische Entfernung. Wegen der lokalen Infiltration in das umliegende Gewebe sollten bei einer Chirurgie ein lateraler Rand von $3 \mathrm{~cm}$ und ein tiefer Rand inklusive Entfernung der Faszie eingehalten werden. Wenn dies nicht möglich ist und 


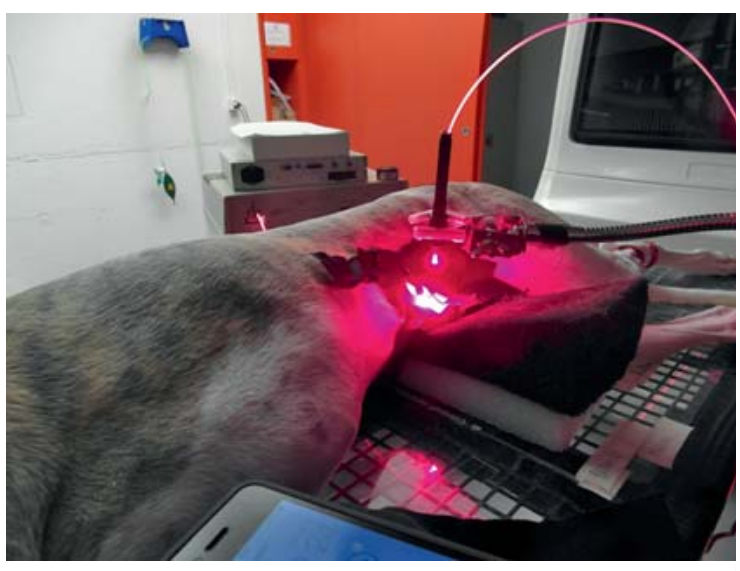

- Abb. 4 Photodynamische Therapie bei einem Patienten mit multiplen oberflächlichen kutanen Hämangiosarkomen. ( ) Julia Buchholz

die Ränder infiltriert sind (mikroskopischer Tumor), spielt die adjuvante Bestrahlung eine wichtige Rolle.

Merke

Eine alleinige Bestrahlung makroskopischer Sarkome ist nicht als kurativ anzusehen, da Sarkome strahlenresistente Tumoren sind.

Zum Teil können anfangs inoperable Sarkome nach neoadjuvanter Bestrahlung operativ entfernt werden ( $\vee$ Abb. 3).

Die konventionelle Chemotherapie nimmt eine untergeordnete Stellung ein. Eine metronomische Chemotherapie kann jedoch sinnvoll eingesetzt werden, z. B. bei Patienten mit inkomplett exzidierten Weichteiltumoren, bei denen eine Strahlentherapie nicht infrage kommt. Dies kann zu einer Verlängerung der Tumorkontrolle führen. Die metronomische Chemotherapie wird oral kontinuierlich durch den Besitzer verabreicht. Sie wirkt über eine Beeinflussung des Immunsystems, Antiangiogenese und Entzündungshemmung. Verwendete Medikamente sind meist Cyclophosphamid und Piroxicam.

Oberflächliche kutane Hämangiosarkome können alternativ zu einer chirurgischen Entfernung (z.B. wenn eine Chirurgie aufgrund der Lokalisation oder Anzahl der Läsionen schwer durchführbar ist) auch erfolgreich mit der photodynamischen Therapie behandelt werden ( $\bullet$ Abb. 4).

\section{Kutane Lymphome}

Das kutane Lymphom lässt sich in 2 Formen unterteilen:

- das nicht epitheliotrope Lymphom

- das epitheliotrope T-Zell-Lymphom
Das nicht epitheliotrope Lymphom kann seinen Ursprung primär in der Dermis haben oder im Zusammenhang eines systemischen Lymphoms auftreten.

Das epitheliotrope T-Zell-Lymphom zeigt sich meist in Form der klassischen Mykosis fungoides. Klinisch aggressiver stellt sich das Sézary Syndrom dar: Hier liegen zusätzlich Blutbildveränderungen (atypische T-Lymphozyten) und z. T. eine generalisierte Lymphadenopathie vor.

Klinik

Die initialen Stadien der Mykosis fungoides ähneln Dermatosen anderen Ursprungs und können sich als Hautrötung teilweise mit Schuppen und später als Plaques darstellen. Pruritus und Sekundärinfektionen können beobachtet werden. Nachfolgend können Hautknoten bzw. Hautmassen auftreten. Bei der „d'emblée“-Variante kann es direkt zur Ausbildung knotiger Veränderungen kommen. Zusätzlich zu den Hautveränderungen können auch mukokutane oder orale Veränderungen vorkommen. Es wird diskutiert, ob ätiologisch chronische Dermatitiden oder Viruserkrankungen eine Rolle spielen.

\section{Diagnostik}

Die Diagnosestellung erfolgt über eine histologische Untersuchung von Punch-Biopsien der veränderten Hautareale. Ein komplettes Lymphom-Staging des Patienten sollte dem Besitzer angeraten werden.

\section{Therapie}

\section{Lokalisierte Formen}

Therapeutisch kommt für lokalisierte Formen eine chirurgische Exzision oder eine Strahlentherapie infrage und dies kann zu langfristiger Kontrolle führen. Da Lymphome sehr strahlensensitiv sind, braucht man i.d. R. keine sehr hohe Totaldosis (das Protokoll besteht aus wenigen Einzelsitzungen). Trotzdem sollten die Besitzer über das mögliche Auftreten weiterer Läsionen aufgeklärt werden.

\section{Multiple Läsionen}

Beim Auftreten multipler Läsionen und bei generalisiertem oder systemischem Geschehen ist die Prognose schlechter und meist leben die Patienten nicht länger als ein Jahr. Beschriebene Therapien für diese Szenarien sind vor allem die Chemotherapie und der Einsatz von Retinoiden oder Kortikosteroiden.

In einer 2018 im Journal of Veterinary Dermatology veröffentlichen Studie, bei der 148 Fälle eingeschlossen wurden, konnte gezeigt werden, dass Hunde mit kutanen Läsionen (vs. mukokutan) und Hunde mit multiplen Läsionen eine schlechtere Prognose haben. Von den 80 Hunden mit kutanen epitheliotropen T-Zell-Lymphomen hatten wiederum die Hunde mit nur einer Läsion sowie die Hunde, die Chemotherapie erhielten, eine bessere Prognose [3]. 
Das am häufigsten eingesetzte Chemotherapeutikum ist Lomustin (CCNU). Auch beschrieben ist der Einsatz von Doxorubicin, L-Asparaginase oder das CHOP-Protokoll. Das höchste komplette Ansprechen ist beschrieben nach dem Einsatz von Lomustin oder liposomalem Doxorubicin: 1/3 der Patienten zeigte eine komplette Tumorremission. Das Gesamtansprechen auf Lomustin (komplette und partielle Remission) ist ca. $80 \%$ und die mittleren Überlebenszeiten nach dem Einsatz von Lomustin sind ca. 6 Monate. Lomustin wird oral mit einer Dosis von $70 \mathrm{mg} / \mathrm{m}^{2}$ Körperoberfläche alle 3 Wochen verabreicht. Es kann zu einer Myelosuppression kommen, die eine Dosisreduktion erforderlich machen kann, sowie zu einer kumulativen Hepatotoxizität und Thrombozytopenie, die ein Absetzen des Medikaments erforderlich machen können. Der Einsatz von Leberschutzpräparaten kann bei erhöhten Leberenzymen zu einer Besserung führen [3].

Bei einigen mit Retinoiden behandelten Hunden sind mittlere Überlebenszeiten von bis zu 11 Monaten beschrieben. Mit Kortikosteroiden behandelte Patienten erreichten mittlere Überlebenszeiten von ca. 4 Monaten [3]. Limitiert sind die Ergebnisse der Studien häufig durch wenige Patienten pro Behandlungsgruppe.

\section{Papillome (Warzen)}

Bei diesen benignen Hauttumoren unterscheidet man 2 Formen:

- die Papillomatose junger Hunde

- einzelne Papillome älterer Hunde

Bei jungen Hunden tritt durch eine Virusinfektion die sogenannte Papillomatose auf. Diese kann sich durch mehrere flache bis gestielte oder blumenkohlartige Umfangsvermehrungen an der Mundschleimhaut, den Augenlidern, Ohren, Gliedmaßen, äußeren Geschlechtsorganen und anderen Lokalisationen äußern. Durch direkten Kontakt zwischen Hunden kann es zu einer Übertragung kommen, bei beeinträchtigtem Immunsystem wird die Entstehung begünstigt. Oft kommt es innerhalb weniger Monate zu einer spontanen Rückbildung der Läsionen. Manchmal ist eine Therapie mittels Autovakzine indiziert.
Beide Formen der Warzen können auch mittels Kryotherapie oder Elektrochirurgie entfernt werden. Bei therapieresistenten Formen der Papillomatose junger Hunde hat die Autorin gute Erfahrungen mit dem Einsatz der Strahlentherapie gemacht: Diese kann unter Umständen auch bei weit fortgeschrittenen Fällen als kurativ angesehen werden.

\section{Fazit}

Die Haut ist eine sehr häufige Lokalisation von Neoplasien beim Hund. Erscheinungsbild und biologisches Verhalten der verschiedenen Hauttumoren sind sehr variabel und die Prognose hängt vom Tumortyp ab. Ein korrektes diagnostisches Vorgehen ist daher entscheidend für die Planung der geeigneten Therapieoptionen.

\section{Korrespondenzadresse}

Dr. med. vet. Julia Buchholz

Dipl. ACVR, Dipl. ECVDI (Radioonkologie)

Tierklinik Hofheim

Katharina-Kemmler-Str. 7

65719 Hofheim

julia_buchholz@yahoo.de

Literatur

[1] Schultheiss PC. Histologic features and clinical outcomes of melanomas of lip, haired skin, and nail bed locations of dogs. J Vet Diagn Invest 2006; 18: 422-425

[2] Wobeser BK, Kidney BA, Powers BE et al. Agreement among surgical pathologists evaluating routine histologic sections of digits amputated from cats and dogs. J Vet Diagn Invest 2007; 19: 439-443

[3] Chan CM, Frimberger AE, Moore AS. Clinical outcome and prognosis of dogs with histopathological features consistent with epitheliotropic lymphoma: a retrospective study of 148 cases (2003-2015). Vet Dermatol 2018; 29(2): 154-159

\section{Bibliografie}

DOI https://doi.org/10.1055/a-0648-2829

Kleintier konkret 2018; 21: 41-48

(c) Georg Thieme Verlag KG Stuttgart · New York ISSN 1434-9132 